\title{
Impaired procedural learning after damage to the left supplementary motor area (SMA)
}

\author{
Hermann Ackermann, Irene Daum, Markus M Schugens, Wolfgang Grodd
}

\begin{abstract}
Two paradigms of procedural learning, serial reaction time and tracking tasks, were given to a patient with damage to the supplementary motor area (SMA) of the left mesiofrontal cortex. This patient exhibited impaired procedural learning during the serial reaction time test and mirror reversed tracking. Unreversed tracking performance was normal. Therefore the SMA-as well as the cerebellum and the striatum-participate in the development of skills requiring internal elaboration of motor behaviour.
\end{abstract}

(F Neurol Neurosurg Psychiatry 1996;60:94-97)

Keywords: procedural learning; implicit learning; supplementary motor area 6

Declarative and procedural-or in more recent terms non-declarative-memory and learning are considered two distinct modes of the acquisition of knowledge. ${ }^{1}$ The first system mediates the long term storage of episodic information which can be retrieved intentionally. Non-declarative memory, by contrast, is characterised by behavioural changes as a result of experience rather than by recollection. A typical example of non-declarative learning is the development of skills. Recently, visuomotor procedural learning was reported to be impaired in patients with cerebellar degeneration or Huntington's disease..$^{23}$ These results are in accordance with theoretical models relating skill acquisition to the basal ganglia and the cerebellum. ${ }^{4}$ Both of these structures are reciprocally connected with the supplementary motor area (SMA) of the mesiofrontal cortex. ${ }^{56}$ Thus the SMA might contribute to the development of skills as well. Positron emission tomography studies, which disclosed SMA activation during motor learning, corroborate this assumption. ${ }^{78}$ On this basis, patients with SMA disorders would be expected to show an impairment in skill learning tasks. However, this deficit has not been shown so far. Therefore, two standard paradigms of visuomotor procedural learning, serial reaction time and tracking tasks, were given to a patient with a circumscribed lesion of the left mesiofrontal cortex after removal of a brain tumour.

\section{Methods}

CASE REPORT

The patient, a 43 year old woman, experienced several generalised nocturnal cerebral seizures. In addition, ictal vocalisations in terms of involuntary repetition trains of the syllable /da/ often occurred. Magnetic resonance imaging carried out after the first epileptic event showed a circumscribed gyral enlargement within the region of the upper left mesiofrontal cortex. Because of the lesion's progression during follow up, a brain tumour was suspected. Apart from word finding difficulties clinical examination had always been unremarkable. Surgical intervention took place about 12 months after the inital seizure. Figure 1 shows the MRI obtained two months before surgery and fig 2 shows the CT obtained one day after the operation. Microscopic evaluation of the removed tissue yielded a diagnosis of glioblastoma. After tumour removal the patient had a paresis of the right leg (which subsided within two weeks) and showed dysfluent hesitant speech. At the time of the present examination, about three months after surgical intervention, postoperative radiotherapy had been completed. The patient had received antiepileptic medication (carbamazepine) since the first generalised seizure.

Neuropsychological assessment after surgery showed average intellectual abilities (IQ = 106; short version of the Wechsler adult intelligence scale). Measures of attention (digit and block tapping spans) were within the normal range. Evaluation of frontal lobe function showed unimpaired performance on the Wisconsin card sorting test whereas two out of three subtests of a German verbal fluency task yielded mild impairments relative to age and IQ matched controls. Formal language testing did not disclose any aphasic deficits (see Daum and Ackermann ${ }^{9}$ ). The patient was right handed and there was no family history of left handedness.

\section{SERIAL REACTION TIME TASKS}

A serial reaction time task was developed in accordance with the procedure described by Knopman and Nissen. ${ }^{4}$ Briefly, a filled circle appeared within one of four squares arranged in a semicircle on the computer screen. The position of the squares corresponded to the locations of raised response buttons on a 
Figure 1 Magnetic resonance imaging in the patient two months before surgery. Lefi: $T 1$ weighted image after contrast media application, FLASH sequence, $T R=30 \mathrm{~ms}$, $T E=12 \mathrm{~ms}$, fip angle $=$ $30^{\circ}$. Right: T2 weighted image, spin echo sequence, $T R=4509 \mathrm{~ms}, \mathrm{TE}=$ contrast enhancing space occupying lesion in the apical parts of the left superior frontal gyrus with compression of the cingulate sulcus. 120 ms. The scans show a

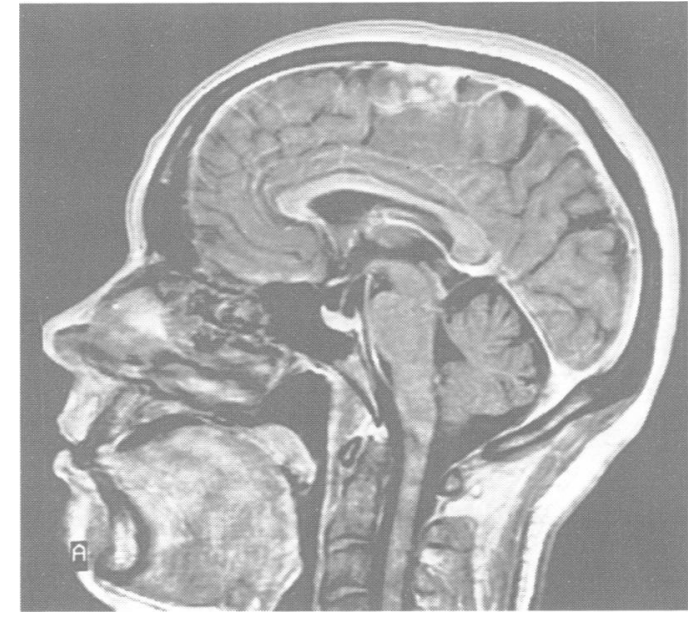

board below the monitor. A fifth button ("home button"), equidistant to the four response buttons, had to be kept pressed between stimulus presentations. Subjects were instructed to release the home button on each trial and to press the response button corresponding to the stimulus position on the screen as quickly as possible. Blocks 1 to 4 and 6 to 8 comprised 110 trials each: the initial five circle positions were in random sequence, followed by 10 repetitions of an identical 10 item sequence ("DBCACBDCBA", denoting the four circle positions from left to right as $\mathrm{A}, \mathrm{B}$, $\mathrm{C}$, and D), concluding with another five randomised stimuli. By contrast, block 5 comprised a random sequence of the 110 circle positions. To assess explicit knowledge of the repetition pattern, a "generate" task was given

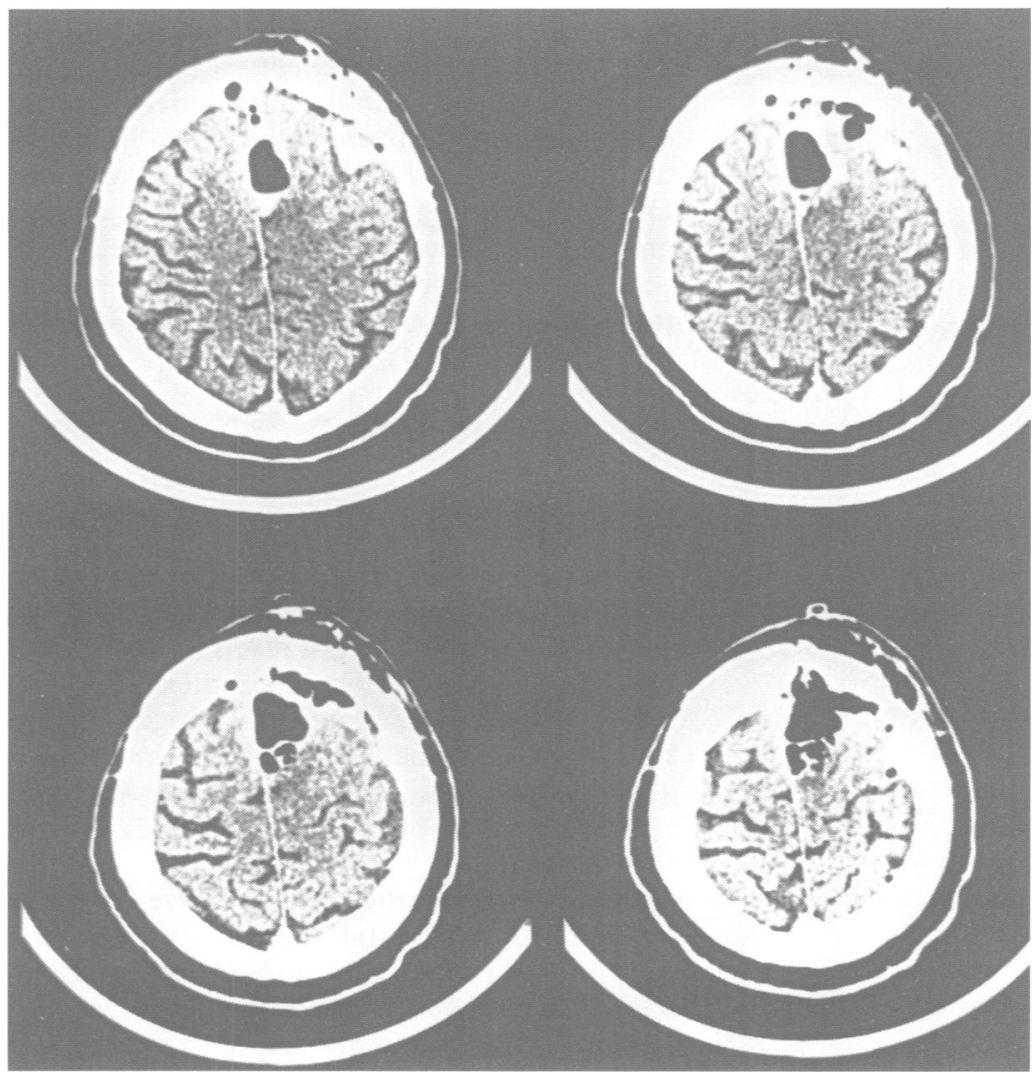

Figure 2 Contrast enhanced CT (consecutive $4 \mathrm{~mm}$ slices) one day after removal of a left mesiofrontal glioblastoma (left hemisphere on the right side of the figure, picture in the bottom right being the most apical). The resection cavity in the left superior frontal gyrus is filled with air. Note local swelling of the adjacent white matter.

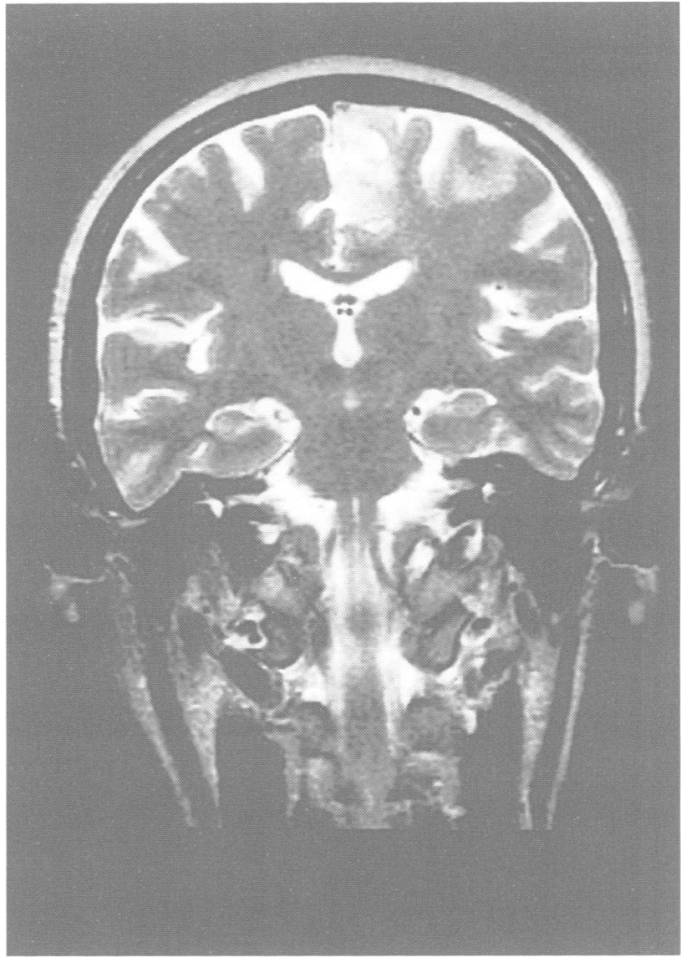

after the last block of trials: subjects had to press the button at the location where they thought the next stimulus would appear in response to 30 sequences of four circle positions each. For each block the median reaction time-the time interval between stimulus appearance and pressing of the respective response button-was computed, excluding the initial and the final five trials which were presented in random order in all blocks.

\section{TRACKING}

Two tracking tasks were adapted from the procedure described by Frith and coworkers. ${ }^{10}$ Subjects had to track a target, a $7 \mathrm{~mm}$ square on a computer screen, by trying to keep a pointer (a cross) within the square using a small $35 \mathrm{~mm}$ joystick. In task 1 the horizontal target movements were determined by a single sine wave of $0.15 \mathrm{~Hz}$ and were, therefore, predictable. The vertical movements, by contrast, reflected a combination of three sine waves $(0.06 \mathrm{~Hz}, 0.11 \mathrm{~Hz}, 0.23 \mathrm{~Hz})$ yielding an unpredictable pattern. During task 2 the target movements were rendered unpredictable in both directions (horizontal: combination of sine waves of a frequency of $0.03 \mathrm{~Hz}, 0.21 \mathrm{~Hz}$, and $0.32 \mathrm{~Hz}$; vertical: $0.05 \mathrm{~Hz}, 0.19 \mathrm{~Hz}$, and $0.33 \mathrm{~Hz}$ ). In addition, the relation between pointer and joystick movements was mirror reversed for the horizontal direction. For each task subjects completed two sessions of three minutes in duration, separated by a resting interval of six minutes. The total time for which they were able to keep the pointer within the target limits was measured for each session. All subjects performed these tests with the right hand.

\section{CONTROLS AND STATISTICAL ANALYSIS}

The control group comprised 15 age matched right handed healthy subjects (mean age $42 \cdot 6$ 
Figure 3 Performance of the patient (unfilled circles) and 15 healthy control subjects (filled circles; group means and $95 \%$ CIs (mean $\pm(S E M \times 2 \cdot 145)(=t$ (14, 0.05))) during the serial reaction time task. Each block comprised 110 trials. For further explanations see text.
Figure 4 Performance of the patient (unfilled circles) and 15 healthy control subjects (filled circles; group means and 95\% CIs (mean \pm (SEM $\times 2 \cdot 145)(=t(14$, $0.05)$ )) during unreversed (upper panel) and mirror reversed tracking (lower panel). The measure "time on target" is displayed separately for the horizontal and vertical direction $(1,2=$ first and second session, respectively).

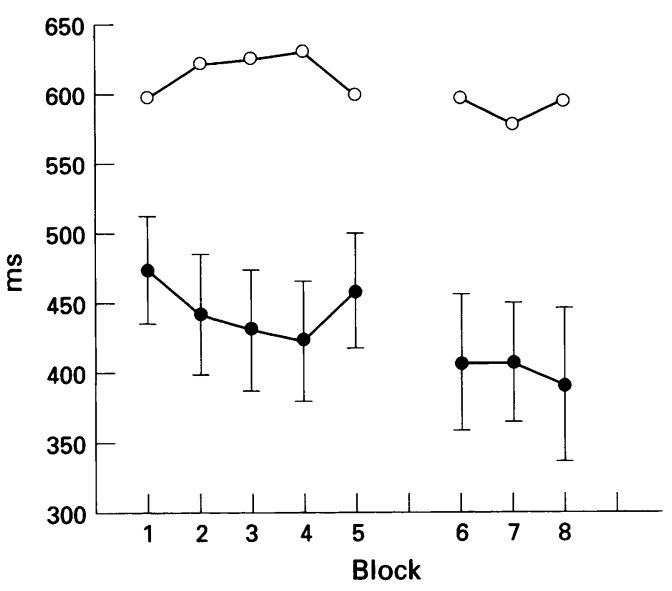

(SD 12.5) years). The results obtained from the patient were compared with those of the control group in terms of " $z$ scores." As the patient was expected to show an impairment in motor skill acquisition, only one sided significance tests were considered.

\section{Results}

SERIAL REACTION TIME TASKS

Figure 3 illustrates the development of reaction time across the different stages of learning.

The decrease of group means in the control subjects across blocks 1 to 4 ( $52 \mathrm{~ms}$, SD 43.0$)$ represents reaction time task learning. By contrast, the patient's reaction time increased by $32 \mathrm{~ms}$. The difference in learning between the patient and the controls achieved statistical
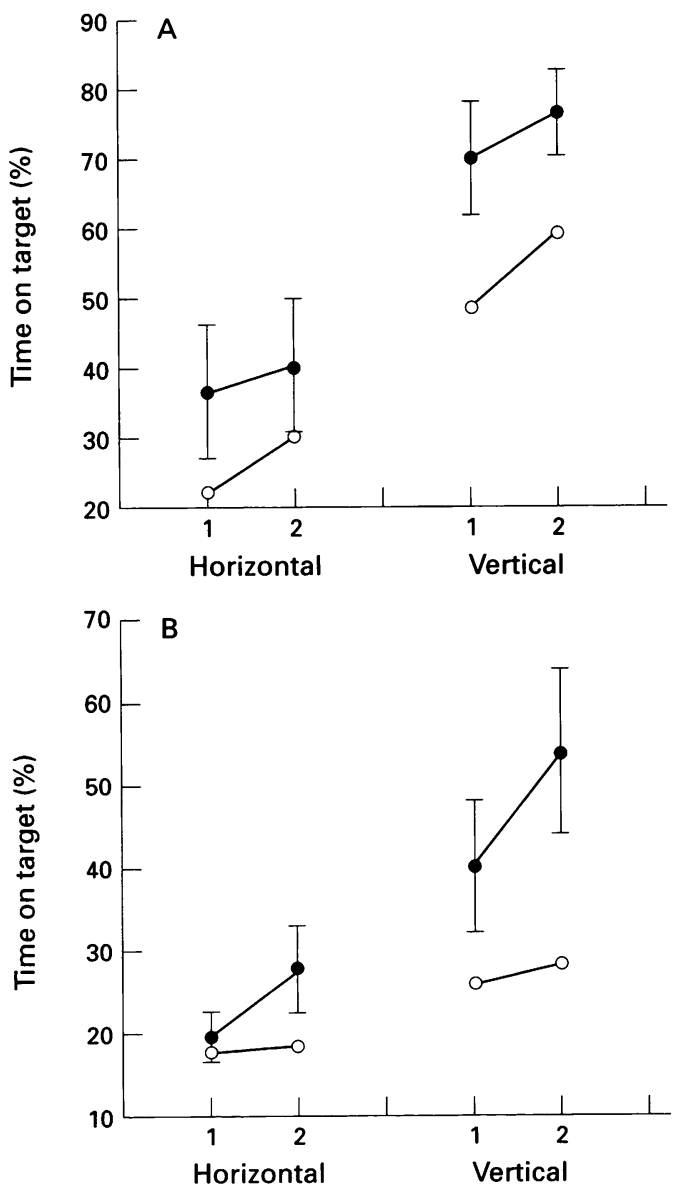

significance $(z=1.95, P<0.05)$. The block 5-block 4 difference, which indicates sequence specific learning, was 36 (SD 24.3) $\mathrm{ms}$ in the control group and $-31 \mathrm{~ms}$ in the patient $(\mathrm{z}=$ $-2.79, \mathrm{P}<0.01)$. During all trial blocks the patient showed significantly slower reaction times than the controls (all $z>1 \cdot 76$, $P<0.05)$. Analysis of retention during reaction time task learning - that is, the median reaction time difference between blocks 4 and 6-did not yield a significant difference between the patient and the control group (controls 15.4 (SD 20.3) ms; the patient: 34 $\mathrm{ms})$. It is doubtful, however, whether the observed median reaction time decrease between blocks 4 and 6 in the patient really reflects retention as there was no evidence of learning across the first four blocks and because the patient's reaction time did not differ between block 1 and block 6 . Performance on the "generate" task which reflects acquisition of declarative knowledge ${ }^{3}$ was intact (controls $56 \cdot 2$ (SD $14 \cdot 8$ ) \% correct; the patient: $50 \%)$.

\section{TRACKING}

In the first task, both the control group and the patient achieved a significant improvement of the "time on target" measure across sessions (fig 4, upper panel).

The between session improvement shown by the patient amounted to $7 \cdot 9 \%$ for the horizontal and $10 \cdot 8 \%$ for the vertical components. These data lay within $1 \mathrm{SD}$ of the control scores (3.7 (SD 4.2)\%, and 6.4 (SD 4.8)\% respectively). For the mirror reversed task, the "time on target" scores of the controls increased by 8.0 (SD 5.0 ) \% and 13.8 (SD $7 \cdot 2) \%$ respectively, between sessions (fig 4, lower panel). By contrast, The patient showed only minimal improvements of $0.8 \%$ (horizontal direction) and $2 \cdot 4 \%$ (vertical direction). Despite the large variance in the control group, the patient's learning scores tended to be significantly poorer than the respective control values $(\mathrm{z}=-1.43$ and $-1 \cdot 60$, respectively, both $\mathrm{P}<0 \cdot 10$ ).

\section{Discussion}

The present study showed impaired procedural learning during serial reaction time and mirror reversed tracking tasks in a patient with damage to the left mesiofrontal cortex. This inability to learn the serial reaction time task is not likely to result from the patient's general N reaction time slowing and initial high reaction time values as the control subjects did not 0 show any significant correlations between reaction time in block 1 and the measures of learning.

As shown by the postoperative CT the patient's mesiofrontal lesion involves the SMA. As visuomotor procedural learning deficits have been found after cerebellar and $\mathbb{D}$ striatal dysfunctions and as both cerebellum and basal ganglia are connected to the SMA, 8 the impairments noted in the patient most likely reflect damage to this circuitry. Admittedly, the patient's paresis of the right 
leg after surgery indicates, in addition, dysfunction of the primary motor cortex which is located posterior to the SMA. Because muscular weakness subsided within about two weeks, significant structural damage to this region can be excluded. Most likely oedema of the medial and apical components of the primary motor area or the respective white matter accounted for this transient deficit. The remaining postoperative clinical sign, dysfluent hesitant speech, is attributable to a disorder of the SMA. ${ }^{11}$ As regards the extent of the postoperative lesion in the ventral direction, the surgical report states that tumour extirpation spared the cingulate gyrus. As there are no distinct neuroradiological landmarks of the anterior borders of the SMA - that is, the transition from the agranular to the dysgranular/granular mesiofrontal cortex-it cannot be excluded that the patient's lesion extended to areas 8 and/or 32 (Brodmann). However, neither clinical nor experimental data indicate any participation of these regions in motor learning. Furhermore, the patient showed normal performance in tasks considered sensitive to dysfunctions of the prefrontal cortex such as the Wisconsin card sorting test and no behavioural signs typically associated with prefrontal damage were seen. Admittedly, the patient presented with slight fluency problems. These disorders might reflect, however, hesitant speech in terms of disturbed initiation of articulatory processes. ${ }^{12}$

By contrast with the serial reaction time and mirror reversed tracking tasks, the patient improved her performance to a similar degree as the controls during unreversed tracking. As the SMA seems to predominantly participate in the initiation of internally generated movement sequences ${ }^{5}$ and as unreversed visually guided tracking does not require significant internal elaboration of motor behaviour, the sparing of this function is not surprising. The serial reaction time and mirror reversed tracking tests, by contrast, should impose higher demands on visuomotor processing such as the sequencing of motor responses or the spatial reorganisation of the visually presented target. Besides the patient's relatively intact neuropsychological status this differential pattern of performance on various motor skill acquisition tasks provides a further argument against the suggestion that the procedural learning deficits are due to radiotherapy or antiepileptic medication. These can be expected to give rise to more general cognitive impairments.

In summary, the present findings confirm previous PET results ${ }^{78}$ indicating the SMA to be a part of circuits mediating visuomotor skill acquisition. Besides the SMA, these "loops" involve the cerebellum and the basal ganglia. The differential contributions of these structures to procedural learning remains to be determined. For example, cerebellar patients show problems in unreversed, but not with mirror reversed tracking tasks. ${ }^{13}$

This study was supported by grants from the German Research Society (Da 259/1-2; SFB 307, B10). We thank Professor J Dichgans for helpful comments on the manuscript.

1 Squire LR. Memory and brain. Oxford: Oxford University Press, 1987.

2 Pascual-Leone A, Grafman J, Clark K, et al. Procedural learning in Parkinson's disease and cerebellar degeneration. Ann Neurol 1993;34:594-602.

3 Knopman D, Nissen MJ. Procedural learning is impaired in Huntington's disease: evidence from the serial reaction time task. Neuropsychologia 1991;29:245-54.

4 Mishkin M, Malamut B, Bachevalier J. Memories and habits: two neural systems. In: Lynch G, McGaugh IL Weinberger NM, eds. New York: Guilford, 1984:65-77.

5 Goldberg G. Supplementary motor area structure and function: review and hypotheses. Behav Brain Sci 1985; 8:567-616.

6 Wiesendanger $M$. Recent developments in studies of the supplementary motor area of primates. Rev Physiol Biochem Pharmacol 1986;103:1-59.

7 Seitz RJ, Roland PE, Bohm C, et al. Motor learning in man: a positron emission tomographic study. Neuroreport 1990;1:57-60.

8 Grafton ST, Mazziotta JC, Presty S, et al. Functional anatomy of human procedural learning determined with regional cerebral blood flow and PET. If Neurosci 1992; 12:2542-8

9 Daum I, Ackermann H. Dissociation of declarative and nondeclarative memory after bilateral thalamic lesions: a case report. Intern $\mathcal{F}$ Neuroscience 1994;75:153-65.

10 Frith $\mathrm{CD}$, Bloxham CA, Carpenter $\mathrm{KN}$. Impairments in the learning and performance of a new manual skill in patients with Parkinson's disease. $\mathcal{F}$ Neurol Neurosurg Psychiatry 1986;49:661-8.

11 Jonas S. The supplementary motor region and speech emission. F Commun Disord 1981;14:349-73.

12 Ackermann H, Hertrich I, Ziegler W, Bitzer M, Bien S. Acquired dysfluencies following infarction of the left Acquired dysfluencies following infarction of

13 Sanes JN, Dimitrov B, Hallett M. Motor learning in patients with cerebellar dysfunction. Brain 1990;113: 103-20. 